\title{
Intermédialités
}

Histoire et théorie des arts, des lettres et des techniques

Intermediality

History and Theory of the Arts, Literature and Technologies

\section{Une ombre dans le regard}

\section{Éric Lecerf}

Numéro 3, printemps 2004

Devenir-Bergson

Becoming-Bergson

URI : https://id.erudit.org/iderudit/1005467ar

DOI : https://doi.org/10.7202/1005467ar

Aller au sommaire du numéro

Éditeur(s)

Centre de recherche sur l'intermédialité

ISSN

1705-8546 (imprimé)

1920-3136 (numérique)

Découvrir la revue

Citer cet article

Lecerf, É. (2004). Une ombre dans le regard. Intermédialités / Intermediality, (3),

31-49. https://doi.org/10.7202/1005467ar
Résumé de l'article

L'une des caractéristiques essentielles de la philosophie d'Henri Bergson tient dans son refus d'entrer dans ce mode de systématisation de la pensée qu'est la dialectique. Au delà de son apport déterminant sur une ontologie dérivée de la durée, ou encore sur l'inscription de l'intuition comme sens initial propre à dé-substantialiser toute forme de dualisme, cet apport singulier permet de comprendre l'intérêt renouvelé pour cette oeuvre qui, de façon exemplaire, réintroduit du mouvement dans un être-pour-la-liberté auquel nulle négativité ne vient conférer sa raison d'exister. Cet article propose de retrouver comment Henri Bergson entreprend de se situer hors de la dialectique en interrogeant le renversement, tout à la fois discret et démiurgique, qu'il effectue du thème de l'ombre. À travers cette réfutation implicite du mythe fondateur de la caverne, nous le voyons ainsi conférer à la vie mécanique sa pleine valeur de vie aliénée. 


\title{
Une ombre dans le regard
}

\author{
ÉRIC LECERF
}

LE PROBLÈME DE L'OMBRE

Présenter les idées implicites sur lesquelles repose la philosophie de Bergson, sans avoir recours aux catégorisations réductrices, devrait constituer un but pour quiconque a porté un minimum d'attention à son œuvre; c'est-à-dire pour quiconque a donné à sa lecture le sens et la valeur d'une expérience. Un but dont on sait néanmoins qu'il demeurera hors de portée, ne serait-ce qu'en raison des limites du langage et des concepts dont l'œuvre de Bergson n'a cessé de montrer les implications, tant concrètes que métaphysiques. Pour pallier cette défaillance, Bergson, qui assume pleinement sa position de philosophe, et ce faisant les limites inhérentes à son propre discours, nous propose d'adopter une attitude singulière: à savoir convertir notre attention. Il s'agit ainsi de "détoumer cette attention du côté pratiquement intéressant de l'univers et de la retoumer vers ce qui, pratiquement, ne sert à rien ${ }^{1}$ ».

De cette attitude, à laquelle Bergson associe explicitement une qualité essentielle de la philosophie, nous tenterons de nous inspirer dans la mesure où notre propos, par son objet même, présuppose une telle conversion. Certes, il serait pour le moins hasardeux de prétendre que la pratique ne confère à l'ombre aucune utilité. Ni d'ailleurs que sa présence, dans le discours philosophique, puisse relever d'une contingence dont la rhétorique serait seule capable de nous livrer les clefs d'interprétation. Origine de la représentation picturale pour les uns ou traduction visuelle de la représentation pour les autres, la présence

1. Henri Bergson, «La perception du changement» [1911], La pensée et le mouvant [1934], dans (Euvres, André Robinet (éd.), Paris, Presses universitaires de France, 1970 [1959], p. 1373-1374. C'est Bergson qui écrit ces deux infinitifs en italiques. 
de l'ombre n'est jamais fortuite, même si l'habitude du regard qui la voit sans la chercher la rend presque invisible. L'attention que le philosophe lui prête ne saurait donc être anodine. Mais encore faut-il qu'il y ait dans cette attention assez de maîtrise pour éviter, comme cela est le cas avec le savant d'Andersen, que l'ombre, livrée à elle-même, ne s'émancipe, mène sa vie et finisse par vouloir se substituer au sujet lui-même². En effet, dès lors que l'ombre échappe au jeu des phénomènes physiques pour investir le discours philosophique, elle admet trois valeurs dont il n'est pas toujours facile de distinguer les implications.

Elle est d'abord une trace de ce qui a été, ou un signe annonciateur de ce qui sera, au sens où Sieyes, par exemple, dans son célèbre Qu'est-ce que le Tiers État?, fait glisser les ombres sur les ordres de représentation politique pour marquer l'empreinte d'un temps que la Révolution est en train d'accomplir. Celui qui va advenir, comme sujet de l'histoire, attend son jour de gloire dans une ombre qui ressemble à s'y méprendre à celle où vient échouer tout sujet dont le temps est déjà révolu. Le $\mathrm{xx}^{\mathrm{e}}$ siècle, dans son souci de mettre en lumière les processus, passera maître dans le croisement infini de ces ombres signifiantes; ombres portées d'un présent qui n'est plus, lui-même, qu'une manifestation des histoires tissées hors de notre détermination à l'habiter.

L'ombre apparaît aussi comme ce refuge auquel la philosophie a eu si souvent recours pour se prémunir contre le ressentiment des puissants et le besoin de croyance des faibles. Des stoïciens jusqu'aux dissidents des crises identitaires contemporaines, l'ombre même du texte permet à la vérité de se frayer un chemin secret qui ne pourra être identifié que par celui qui acceptera de l'expérimenter. Trop bien constituée, elle tend cependant à se transformer en une clôture, un espace trop bien conçu pour qu'il soit encore possible d'y

2. Dans le conte intitulé «L'ombre», «un savant du Nord» épuisé par son séjour sous le soleil africain, laisse échapper son ombre afin qu'elle épie une belle femme (la poésie) qu'il n'a fait qu'entrevoir. Lorsque, plusieurs années plus tard, l'ombre réapparaîtra, elle renversera les rôles et fera du savant son ombre, jusqu'au jour où, pour accéder au pouvoir, elle le fera mettre à mort. Voir Hans-Christian Andersen, L'ombre et autres contes, trad. Marc Auchet, Paris, Librairie générale française, coll. «Libretti », 2001 [1847].

3. Notamment lorsqu'il écrit: «Il [le Tiers État] ne doit pas ignorer qu'il est aujourd'hui la réalité nationale dont il n'était autrefois que l'ombre; que pendant ce long changement, la noblesse a cessé d'être cette monstrueuse réalité féodale qui pouvait opprimer impunément, qu'elle n'en est plus que l'ombre, et que vainement cette ombre cherche-t-elle encore à épouvanter une nation entière. » (Emmanuel Joseph Sieyès, dit l'Abbé Sieyès, Qu'est-ce que le Tiers-État?, Paris, Éditions de la Société de l'histoire de la révolution française, 1888 [1789], p. 48-49) 
échapper. Labyrinthe ou palais des illusions, l'ombre devient ce lieu où croît l'inconscient jusqu'à perdre l'idée du corps qui, comme chez Jung, tend à n'être plus qu'un outil voué à «personnifier notre ombre ${ }^{4}$ ». La lumière n'est plus ici la substance du présent, mais l'expression d'un interdit vers lequel l'ombre nous renvoie sans cesse. Source de créativité ou d'obsession, elle nous soumet l'hypothèse de cette pleine lumière comme d'un danger que ni l'individu ni la communauté ne pourrait librement fréquenter sans courir le risque d'y être consumé.

L'ombre est enfin, dans sa version dialectique, la source d'un problème. Elle n'est pas simplement ce qui accompagne la lumière, mais elle la conditionne, la limite et, pour finir, la tourmente. La lumière, dans laquelle s'expriment les conditions nécessaires à l'acquisition de la vérité, y apparaît ainsi tout autant comme ce qui surgit d'une ombre que comme un agent producteur d'ombres. Comme l'écrit Peter Sloterdijk, dans le prolongement des thèses adorniennes sur la modernité: «L'Aufklärung ne pénètre pas simplement dans la conscience sociale comme porteuse de lumière sans problème. Là où elle exerce son action, naît une "pénombre", une ambivalence profonde5. »

L'ombre n'est plus un contrechamp du présent ni un refuge mais l'expression signifiante de la lumière, une sorte de révélateur exclusif. Elle se constitue ainsi en problème dans la mesure où, si toute lumière est portée par l'intention de dissiper les ombres, elle ne cesse d'en produire de nouvelles. C'est précisément sur cette désignation d'un «problème » que portera notre lecture de l'œuvre d'Henri Bergson car, au regard de son souci de refonder la métaphysique, non seulement ce problème de l'ombre ne s'impose pas, mais il permet, par son absence même, de comprendre ce qui la distingue des philosophies qui lui sont contemporaines. D’une façon plus décisive encore, la place réduite que Bergson a réservé à l'ombre témoigne de l'écart qu'il a tenté de marquer vis à vis de la dialectique hégélienne et de ses innombrables métamorphoses, des plus simples arrangements jusqu'aux plus rigoureuses réfutations ${ }^{6}$. À travers les divers

4. Carl Gustav Jung, L'homme à la découverte de son âme: Structure et fonctionnement de l'inconscient, trad. Roland Cahen, Paris, Éditions Albin Michel, 1987 [1928], p. 121.

5. Peter Sloterdijk, Critique de la raison cynique, trad. Hans Hildenbrand, Paris, Éditions Christian Bourgois, 1987 [1983], p. 48.

6. L'absence de tout commentaire sur Hegel dans le chapitre IV de L'évolution créatrice est à cet égard particulièrement significative. Voir Henri Bergson, L'évolution créatrice [1907], dans Euvres, p. 725-807. 
plans de questionnement où nous retrouverons les rares ombres que Bergson a laissé se faufiler sous sa plume ${ }^{7}$, il apparaît qu'aucune d'entre elles ne pourrait se rapporter à une négativité plus ou moins signifiante. Comme s'il y avait, dans cet usage de l'ombre, de quoi corrompre tout problème et tirer la pensée vers cette impuissance dont témoigne ici Eduard Bernstein, à travers sa célèbre critique de l’hégélianisme de Marx: "[La dialectique hégélienne] ressemble à la vérité comme un feu follet à l'éclairage. Elle ne se contredit pas, puisque d'après elle, chaque chose comporte son antithèse propre ${ }^{8}$.»

L'ombre y occupe la fonction de "principe premier», au sens aristotélicien du terme, puisque c'est en elle que toutes les formes de lumière trouvent de quoi fonder leur unité principielle, jusqu'à ce point extrême où, comme en vient d'ailleurs à le déplorer Bernstein, la dialectique parvient à incarner, à elle seule, la réalité, en raison même de la logique infaillible qui la soutient. Et c'est bien en cette sorte de désappropriation logique de la réalité que ce "problème de l'ombre» déborde la physique pour marquer de son sceau l'existence humaine, des derniers replis de notre intimité jusqu'à cette expression narrative, et quelques fois épique, de la vie communautaire, que nous avons appris à nommer l'histoire. Et ceci, sans même que soit rendu nécessaire un usage métaphorique de ces termes, tant il est vrai que cette opposition entre la lumière et l'ombre semble à la source de nombre de ces antinomies, plus ou moins structurantes, dont notre culture, au sens le plus large du terme, n’a cessé de s'entretenir. La relation entre l'ombre et la lumière constitue à cet égard un exemple particulièrement intéressant de cette inversion des sens dont parle Bergson lorsqu'il écrit que: "Il y a des cas où c'est le langage imagé qui parle sciemment au propre, et le langage abstrait qui parle inconsciemment au figuré 9 .

Du mythe platonicien de la caverne jusqu'à la figure romantique de l'homme sans ombre ${ }^{10}$, la philosophie a été, plus qu’à son tour, mobilisée afin d'établir

7. Nous en avons retiré tous les usages convenus tirés d'expression telles que «laisser dans l'ombre», ou encore le «lâcher la proie pour l'ombre», emprunté aux fables d'Ésope, expression que Bergson semblait assez apprécier pour la placer dans presque tous ses textes.

8. Eduard Bernstein, Socialisme théorique et social-démocratie pratique, trad. Alexandre Cohen, Paris, Éditions Stock, 1900 [1899], p. 63.

9. Henri Bergson, «Introduction (première partie): Croissance de la vérité. Mouvement rétrograde du vrai » [1922], La pensée et le mouvant, p. 1285.

10. Reprise du célèbre récit d'Adalbert von Chamisso, Peter Schlemihl, trad. Hippolyte von Chamisso, Paris, Éditions José Corti, 1989 [1814]. 
le fondement rationnel de la métaphore; c'est-à-dire en vue de reproduire les mécanismes propres à ce simple phénomène physique pour donner à voir et à comprendre comment notre conscience serait elle-même configurée dans une composition identique, jusqu'au point de nous laisser penser que cette configuration-là pourrait nous servir de modèle afin de concevoir comment lumière et ombre parviennent à mettre en relief l'horizon. En effet, si dans les deux termes d'une métaphore on ne sait jamais vraiment quel est celui qui donne du sens à l'autre, l'introduction du couple lumière et ombre dans les choses de l'esprit introduit dans notre perception de l'influx lumineux une valeur symbolique dont celle-ci ne saurait s'émanciper sans avoir effectué un travail préalable de réconciliation avec une "pure expérience» dont nous parlerons plus loin. L'œuvre de Turner, qui a impressionné Bergson, et notamment l'ensemble des tableaux qu'il a pu peindre dans les dix dernières années de sa vie, et ceci quel que soit le motif invoqué pour chacun, témoigne de façon à proprement parler éblouissante de ce travail de réconciliation envers un influx lumineux dépouillé des aléas de la représentation.

Mais avant d'en venir à la représentation, appliquons-nous déjà à voir ce qui, selon Bergson, échappe naturellement au problème dans ce phénomène de l'ombre; «en clair», ce qui relèverait du «propre» de l'ombre. Car si ce problème de l'ombre n'a de sens que dans la conjonction d'une irréductible insolvabilité et d'une déroutante simplicité, c'est d'emblée vers cette seconde proposition que nous entraînera Bergson.

\section{LE PROPRE DE L'OMBRE}

Pour commencer, nous pourrions dire que le sens commun, si souvent convoqué par Bergson, au point d'accéder parfois au rang de critère de vérité, ne saurait rencontrer la moindre difficulté pour trancher cette question. La lumière et l'ombre vont forcément de pair. Elles ne sont pas simplement complémentaires, mais sont consubstantielles et participent d'une même nature, lors même que l'opposition formelle dont elles sont les vecteurs prioritaires nous permet de structurer une part essentielle de notre perception. La lumière et l'ombre participent d'une connexion première établie entre l'extériorité de cette perception (la perception est, chez Bergson, une émanation de l'objet avant d'être une captation du sujet) et l'intériorité de la sensation (c'est dans la sensation que le sujet est convoqué). On pourrait dire que l'influx lumineux, dont l'ombre n'est jamais qu'une partie constituante, est le vecteur temporel par lequel l'objet, comme image, parvient jusqu'à nous et finit par se construire un 
refuge dans notre mémoire. Par cette connexion, l'unité de la nature nous apparaît comme une suite d'oppositions, dont le couple de la lumière et de l'ombre ne constitue que le premier terme. Celui-ci va nous servir à fragmenter la nature, et ce faisant, à l'intégrer à notre propre nature. C'est ce que tend à démontrer Bergson dans le premier chapitre de l'Essai sur les données immédiates de la conscience ${ }^{11}$, où le partage entre lumière et ombre lui sert à introduire sa célèbre distinction entre l'extensif et l'intensif. Prenant l'exemple de la feuille blanche derrière laquelle on allume ou éteint des bougies afin de faire varier l'intensité lumineuse, il vise ainsi à retrouver le chemin emprunté par la sensation, autrement dit comment l'objet, par la perception, nous affecte au point de concevoir ce qui lui est, à lui, parfaitement étranger, à savoir ces principes contraires dont nous nous servons pour établir des graduations ${ }^{12}$. Dans cette première étude de la relation entre la lumière et l'ombre, nous retrouvons déjà posé l'un des postulats décisifs de la philosophie bergsonienne: l'essence de la pensée humaine est de bâtir un édifice conceptuel stable sur une réalité qui est, quant à elle, toujours mouvante. Action qui, dans ce cas particulier, consiste à fixer des étapes dans ce pur mouvement que constitue l'influx lumineux. De la même façon, lorsque Bergson entreprend, à plusieurs reprises, de définir comment s'agence notre sensation des couleurs, il rapporte directement ce pur mouvement à une durée qui, si elle est imperceptible à nos sens, n'en est pas moins aussi réelle que n'importe quelle autre de ces durées dont nous croyons tout savoir dès lors que nous pensons les avoir soumises à un quelconque étalonnage.

Sur ce premier plan du «propre» de l'ombre, que nous pourrions aussi nommer plan physique (si cette dénomination n'en proposait pas une visée réductrice), l'ombre ne saurait être définie comme une négation, ni même comme une limitation, voire même comme un reflet. Ce qui, en soi, présente une cohérence évidente avec la refondation vitaliste de la métaphysique que n’a cessé de défendre Bergson. La négation n’est pour lui ni un retranchement, ni une fondation, mais toujours un ajout. Néant, désordre, abîme de l'être, ne sont que des «fantômes ${ }^{13}$ » dont le caractère obsessionnel n'est effectif que chez

11. Henri Bergson, Essai sur les données immédiates de la conscience [1889], dans Cuvres, p. 36-41.

12. Graduations qui reposent elles-mêmes sur un étalonnage des causalités, la comparaison directe des sensations étant impossible.

13. Henri Bergson, «Introduction (deuxième partie): De la position des problèmes» [1922], La pensée et le mouvant, p. 1303. 
celui qui tourne le dos à la vie, c'est-à-dire celui qui vit dans l'illusion — ou le désir - de ne pas vivre sa propre vie. L’ombre n’apparaît pas ici comme un négatif, mais elle constitue un élément positif du savoir. Elle est l'instrument dont se sert, par exemple, Thalès, lorsqu'il entreprend à l'aide de sa seule canne de mesurer la hauteur d'une pyramide; ou encore son disciple et successeur Anaximandre, qui, pour imprimer une marque sur la passe du temps, se sert, lui aussi, de l'ombre d'un bâton, passé à la postérité sous le nom de gnomon. L'ombre ne saurait constituer un négatif pour la simple raison qu'elle est à ce point intégrée à l'image qu'elle en devient transparente. Comme l'écrit Bergson dans Matière et mémoire, dès lors qu'on délaisse l'étude des systèmes philosophiques pour s'intéresser au réel de la vie, «la photographie du tout y est translucide [car] il manque, derrière la plaque, un écran noir sur lequel se détacherait l'image ${ }^{14}$. »

Le négatif, version dialectique de cet écran noir photogénique, ajoute toujours au réel quelque chose de trop, une sorte de surdétermination qui, si elle convient parfaitement pour construire un système, enfouit le réel, lumière et ombre confondues, sous une coloration nouvelle, ne serait-ce qu'en raison de la temporalité dont il use pour advenir. Et si le passage par le négatif devait s'avérer indispensable pour qu'advienne une mise en lumière du monde, ce qui sous-entend l'hypothèse d'une ombre inaugurale, celui-ci ne saurait être recherché ailleurs qu'en nous-mêmes. Ce qui donc nous amènera à nous placer sur un second plan qui sera celui de la représentation, ou de ce que nous pourrions nommer le «figuré» de l'ombre. Mais avant d'en venir là, il nous faut en finir avec ce premier plan du «propre» de l'ombre et voir comment, chez Bergson, s'effectue le passage de l'un à l'autre de ces plans, c'est-à-dire de déterminer quel instrument servira de médiateur entre le propre et le figuré.

Pour concevoir ce que peut être la portée d'une ombre, il faudrait sans nul doute être capable de s'en remettre à ce que Bergson nomme à plusieurs reprises une «expérience pure», c'est-à-dire une expérience délivrée de tout souvenir, ou, si l'on préfère s'en remettre à la philosophie kantienne, délivrée de tout a priori. Forts de cette naïveté, nous serions à même de remarquer ce que nous avons cessé de voir, par exemple, l'ombre qui accompagne notre main lorsque nous écrivons. Il y a ainsi dans la considération du «propre » de l'ombre quelque chose d'enfantin qui, précisément ne saurait être une réminiscence

14. Henri Bergson, Matière et mémoire. Essai sur la relation du corps à l'esprit [1896], dans Euvres, p. 188. 
d'un passé plus ou moins lointain, mais à l'inverse apparaît comme la renaissance d'un sensible épuisé dans la nécessaire et constructive représentation ${ }^{15}$. L'expérience pure se définit effectivement, d'abord, comme une expérience de l'instant présent qui échappe de façon absolue au souvenir de toute expérience passée. À ce titre, nous pourrions dire qu'elle induit la capacité de voir pour la première fois ce que nous avons toujours eu devant les yeux. Cette «nouvelle enfance ", qui introduit d'ailleurs l'Essai sur les données immédiates de la conscience - et donc l'œuvre de Bergson - n'est nullement une fin en soi ou un projet existentiel. On pourrait y déceler une certaine connivence avec l'ignorance que Pascal nous enjoint de reconstituer après avoir traversé l'expérience du savoir. Cette expérience pure, dont nous verrons plus loin la dimension esthétique essentielle, s'apparente plutôt à une sorte de limite idéale, un point extrême sur lequel nous ne pourrions pas nous tenir mais qui néanmoins ouvre à la réflexion une perspective de recherche. C'est notamment sur elle que Bergson s'appuie pour établir ses propres définitions de ce fond d'écran sur lequel la représentation vient imprimer sa marque, un fond d'écran dont il nous livre le nom, à travers cette définition devenue canonique: "J'appelle matière l'ensemble des images, et perception de la matière ces mêmes images rapportées à l'action possible d'une certaine image déterminée, mon corps ${ }^{16}$. "

Ce corps, dont il nous dit, quelques lignes plus haut, que nous dessinons les actions virtuelles «à la manière d'une ombre». On pourrait ainsi dire que s'il y a un propre de l'ombre dans Matière et mémoire, c'est bien celui-ci, à savoir ce corps dont nous conjuguons la temporalité dans l'immédiateté de son futur. Cette ombre-là nous devance encore et toujours car notre corps est, par définition, perpétuellement à la traîne, en retard du mouvement que l'ombre, ce corps intérieur que nous sentons, a déjà effectué. Cette ombre est toujours devant nous, tandis que la réalité, pour reprendre les termes employés près de quarante ans plus tard dans La pensée et le mouvant, "projette derrière elle son ombre dans $[\mathrm{ce}]$ passé indéfiniment lointain » où le possible - et non plus le

15. Un enfantin dont témoigne, par exemple, Isaac Bashevis Singer, lorsque dans Le manoir, il écrit d'un de ses personnages qui, pour la première fois de sa vie, est soumis à une passion amoureuse : «[...] une seule fois elle avait connu pareille sensation. C'était pendant son enfance. Elle avait joué à cache-cache avec son ombre sur le mur et l'ombre s'était détachée et l'avait giflée. » (Isaac Bashevis Singer, Le manoir, trad. Gisèle Bernier, Paris, Éditions Stock, 1968 [1967], p. 120)

16. Henri Bergson, Matière et mémoire, p. 173. 
virtuel - trouve son siège ${ }^{17}$. Ces deux ombres, reflet pour la première et sillage pour la seconde, entretiennent entre elles une relation qui n'est en aucune façon dialectique puisque, si l'une trouve de quoi renforcer sa propre empreinte en l'autre, elles sont de nature distincte, notamment en ceci que chacune témoigne d'une modalité d'expression de liberté se situant sur des plans différents. Mais c'est cependant bien dans le croisement de ces deux ombres, ombre du corps qui nous précède et ombre de la réalité qui effectue en nous ce mouvement rétrograde, que nous effectuons nous-mêmes le passage entre le propre de l'ombre et son figuré ou, pour reprendre la thématique bergsonienne, entre la sensation et la représentation.

\section{L'OMBRE AU FIGURÉ}

De ceci, nous pourrions déjà conclure que l'ombre ne saurait constituer un problème, pour peu qu'on lui assigne sa place. C'est un précepte que la vie aurait d'emblée compris, la conscience intégré. Seule la rationalité, au nom d'une théorisation du réel, se serait embourbée dans un mode d'opposition qui nous mettrait à distance de ce réel fuyant plus qu'il ne nous permettrait de l'appréhender. En rester là reviendrait de facto à accorder un certain crédit aux accusations d'antirationalisme dont a si souvent été l'objet Bergson, accusations qui ne sauraient tenir bien longtemps dès lors qu'on prend le risque de sortir des abrégés pour se colleter directement aux textes ${ }^{18}$. Ce sera donc dans sa critique de la négativité que Bergson s'opposera à la plupart de ses contemporains. Nous pourrions dire que ce qu'il réfute, au delà du dogmatisme inhérent à toute forme de systématisation qui ne s'assume pas d'emblée comme fiction ${ }^{19}$, c'est bien la dialectique. En effet, ce que Bergson critique dans la dialectique, p. 1264 .

17. Henri Bergson, «Introduction (première partie)», La pensée et le mouvant,

18. On pourrait à cet égard constater une certaine convergence entre la position de Bergson et la critique de la mythification du rationalisme opérée par Adorno et Horkheimer. En effet, au delà des nombreuses divergences entre les initiateurs de l'école de Francfort et le bergsonisme, notamment sur la question cruciale du négatif, on retrouve néanmoins ce prédicat commun d'une rationalité qui ne peut accéder à sa plénitude qu'en s'interrogeant de façon préalable sur ses propres limites. Voir Max Horkheimer, Theodor W. Adorno, La dialectique de la raison: fragments philosophiques, trad. Éliane Kaufholz, Paris, Éditions Gallimard, coll. «Tel», 1974 [1944].

19. Thème récurrent de la philosophie de Bergson, dont la première page de $L a$ pensée et le mouvant témoigne excellemment lorsqu'il critique ces systèmes qui pour- 
ce n'est pas son côté fictionnel ou systématique, dont il reconnaît qu'il confère aux mathématiques une vertu particulière, mais sa prétention à incarner la vie. Nous en avons un exemple lorsque, dans L'évolution créatrice, aux détours d'une critique des systèmes philosophiques qui, par définition, sont contraints de tout embrasser du monde dans leur propre projection, Bergson témoigne du peu d'intérêt que présente pour lui l'allégorie platonicienne de la caverne. Le commentaire est d'ailleurs assez bref. En fait, il se limite à dire que l'intelligence humaine, productrice du fameux homo faber, n'a pas plus pour fonction de «regarder passer des ombres vaines que de contempler, en se retournant derrière elle, l'astre éblouissant ${ }^{20}$.»

En vérité, que remet-il en cause dans cette figure, dont le caractère fondateur pour la philosophie paraît difficilement contestable? Simplement que le partage opéré ici par Platon n'est qu'une fiction. Non pas la situation ellemême dont Socrate reconnaît sans difficulté le côté pour le moins improbable, mais la représentation de la connaissance que l'allégorie induit. À en croire Platon, il y aurait, d'un côté, ceux, nombreux, qui regardent passer les ombres, et n'ont même d'autre connaissance d'eux-mêmes, de leur propre corps, que la vision de cette ombre qui leur est opposée; de l'autre, une minorité de visionnaires (au sens propre du terme) qui parviendraient à avoir une pleine vision du réel en lui-même. Pour Bergson, ce partage n'a, à proprement parler, aucun sens, en premier lieu, en raison de la confusion entre perception, sensation et représentation qu'il présuppose, mais surtout, comme il l'écrit, car «[l]e monde où nos sens et notre conscience nous introduisent habituellement n'est plus que l'ombre de lui-même ${ }^{21}$. »

Si ce monde n'est que l'ombre de lui-même ou encore, comme Bergson l'ajoute ensuite, «froid comme la mort», cela ne tient certes pas à une quelconque négativité, mais au contraire au fait que «tout y est arrangé pour notre plus grande commodité22.»Autrement dit, ce qui se joue dans ce figuré de

raient tout aussi bien fonctionner pour le cas « où [les hommes] naîtraient décrépits pour finir nourrissons; où l'énergie remonterait la pente de la dégradation; où tout se tiendrait à rebours et fonctionnerait à l'envers. » (Henri Bergson, «Introduction (première partie), La pensée et le mouvant, p. 1254)

20. Henri Bergson, L'évolution créatrice, p. 657.

21. Henri Bergson, «L'intuition philosophique» [1911], La pensée et le mouvant, p. 1364 .

22. Henri Bergson, «L'intuition philosophique», p. 1365. 
l'ombre, c'est notre prise de possession, positive, d'un réel toujours fuyant; c'est l'habitude consommée de ce geste sur lequel nous croyons précisément n'avoir plus rien à dire ni à penser. Car l'ombre elle-même ne révèle rien, sinon, une fois encore, sur nous-mêmes, c'est-à-dire sur notre capacité à produire des images puis surtout à les investir d'un sens dont nous dirons, suivant l'usage que nous en aurons, qu'il est positif ou négatif.

Pour Bergson, un fait, ce n'est jamais qu'un arrangement. La négativité qui lui est rapportée n'est elle-même qu'une composante de cet arrangement, et en aucune façon un révélateur. Ce que dans l'avant-propos de Matière et mémoire, il nomme «le champ clos de la dialectique pure ${ }^{23}$ ", auquel il oppose «l'attention à la vie», s'apparente dès lors à une science des ombres recomposées, de ces «obscurités artificielles» que dans cette même page il nous dit vouloir, sinon dissiper, tout au moins réduire. Et lorsque dans «L'intuition philosophique », il nous engage à "regarder une ombre pour deviner l'attitude du corps qui la projette ${ }^{24}$ », ce n’est pas le réel dans son hypothétique négativité qui est visé, mais la connaissance d'une attitude, en l'occurrence l'attitude du philosophe vis-à-vis de ses propres intuitions. Ce que l'ombre révèle, c'est l'empreinte d'une action, une composition arbitraire à propos de laquelle Bergson n'exprime aucune sorte de condamnation morale, dans la mesure où c'est par cette série de compositions que chacun d'entre nous accède à la position de sujet. Encore faut-il disposer du minimum d'attention nécessaire pour distinguer cette ombre, notamment lorsqu'elle nous est la moins perceptible, c'est-à-dire lorsqu'elle expose l'attitude qui est nôtre pour gérer ce que nous pourrions nommer le cours normal du temps, ce temps ordinaire ou mécanique dont il est si difficile de tirer expérience. Car c'est bien là que réside toute la difficulté, à savoir, comme l'écrit Bergson dans L'énergie spirituelle: «La conscience ne l'aperçoit pas d'ordinaire ${ }^{25}$, pas plus que notre œil ne verrait notre ombre s'il l'illuminait chaque fois qu'il se tourne vers elle ${ }^{26}$.»

Comme nous le verrons plus loin, seule, cette forme singulière d'attention à la vie qu'est l'œuvre d'art peut dépasser cette difficulté. Encore lui faut-il se saisir de cette ombre comme d'une partie intégrante de la perception pure et

23. Henri Bergson, Matière et mémoire, p. 167.

24. Henri Bergson, «L'intuition philosophique», p. 1347.

25. Il s'agit ici du souvenir omniprésent dans la traduction de la simple perception en expérience.

26. Henri Bergson, L'énergie spirituelle [1919], dans (Euvres, p. 913. 
se défaire des habitudes techniques dont la représentation l'a dotée et qui lui font courir le risque de ne plus être qu'un simple déploiement de mécanismes, fussent-ils les plus performants, autrement dit, les plus propres à user de l'ombre comme d'un outil conventionnel de perspective.

Pour en revenir à ce partage implicite entre objectivité et subjectivité qu'induit cette figure des ombres, il ne saurait dès lors avoir de sens dans la philosophie de Bergson puisque le dualisme auquel lui se réfère est celui de la perception et de la représentation, c'est-à-dire de deux actions, et non pas de ces deux états (objectivité et subjectivité) qui nous renvoient de façon quasi automatique vers un jugement moral préalable. Vis-à-vis de ces deux actions, qui sont néanmoins aussi contraires que peuvent l'être dans leur registre l'objectivité et la subjectivité, la philosophie n'a pas à effectuer de choix, et encore moins de tenter d'effectuer entre elles une quelconque synthèse. En effet, cette synthèse entre la perception, qui nous vient de l'objet, et la représentation, qui nous vient de la conscience, n'a nul besoin de la philosophie pour s'effectuer en chaque instant dans ce présent continu que traverse, et surtout qu'exprime, notre corps. Elle lui est naturelle, immédiate et continue. La fonction de la philosophie, ce sera donc plutôt d'en retrouver les éléments purs, de les déconcilier, notamment afin de déterminer les champs d'action de la seule ombre qui lui importera de poursuivre: à savoir ni le propre ni le figuré de l'ombre, mais l'ombre du regard, car c'est en elle que le réel peut être vraiment décrypté.

\section{L'OMBRE DANS LE REGARD}

Le regard, outil de la représentation, est porteur d'ombre. Il n'ajoute pas au réel quelque chose de plus, mais en retranche l'essentiel. Il en éclaire une part, et ce faisant, dépose une ombre sur les parties de ce réel vis à vis desquelles il ne se connaît pas d'utilisation ${ }^{27}$. Là non plus, on ne trouvera pas chez Bergson la moindre trace d'une quelconque critique vis-à-vis de cette attitude qui nous est, à tous, commune. Le regard, porteur d'ombre, se limite à remplir la fonction pour laquelle il a été formé. Nous pourrions même dire que ce qui fait l'essence du regard, c'est précisément sa capacité à introduire des ombres dans un horizon où la pleine lumière, constante et extensive, fût-elle d'ailleurs la lumière infinie de la nuit, doit toujours être réduite, parsemée d'ombres, pour être 
décryptée. Là où s’impose la critique de Bergson, comme nous l'avons déjà vu pour la dialectique, c'est dans notre constante volonté de croire que les ombres ainsi composées par le regard peuvent s’identifier au réel. Ou, plus grave encore, dans la volonté de conclure que le réel n'a pas d'existence en soi dès lors que cette illusion est devenue impossible. Du moins, cette critique ne porte que sur la philosophie et la science, et non pas sur nos gestes et nos regards du quotidien qui, que nous le voulions ou non, sont toujours empreints de cette illusion. «Est-il étonnant, écrit-il à ce propos, [que la science qui a rompu avec l'intuition] voie cet objet fuir devant elle, comme l'enfant qui voudrait se fabriquer un jouet solide avec les ombres qui se profilent le long des murs ${ }^{28}$. »

Qu'il y ait une vertu secrète dans le geste de cet enfant dont, sur le plan esthétique, il semble évident qu'un Christian Boltanski ${ }^{29}$ pourrait se revendiquer, c'est là un point sur lequel nous reviendrons plus loin. Pour l'instant, ce qu'il importe de comprendre c'est que, si l'objet ici en question est celui dont on croit avoir retrouvé la substance en conciliant tous les points de vue émis à son endroit, l'ombre fuyante de cet objet porte, quant à elle, un nom qui nous éloigne encore plus du geste enfantin puisqu'il s'agit du concept. Et cette foisci, pour se perdre dans ce jeu des ombres, il n'est nul besoin, comme dans la caverne de Platon, d'être enchaîné, pas même par le fil de soie des belles sentences et des opinions recommandables. Il suffit de croire que l'expression du concept permet de concevoir le réel dans son intégralité pour errer dans «l'ombre des problèmes infondés ou mal posés ${ }^{30}$.»

La question pourrait dès lors se poser en ces termes: en est-on fatalement réduit à devoir choisir entre un savoir qui ne s'exprime pas, et donc ne se partage pas, et le partage d'un savoir qui n'est jamais qu'une illusion? Pour reprendre, et inverser, l'inquiétude propre au conte d'Andersen, on pourrait se demander si le problème avec l'ombre, ce n'est pas qu'elle s'accompagne toujours de lumière? C'est-à-dire d'un savoir fuyant qui nous embrasse et nous

28. Henri Bergson, «Introduction à la métaphysique » [1903], La pensée et le mouvant, p. 1406.

29. Notamment son installation de 1986, exposée à Paris, intitulée Les ombres.

30. Cette version-ci de l'ombre, pleinement métaphorique, fait elle aussi occurrence dans les textes de Bergson et, notamment, dans les deux premiers chapitres de $L a$ pensée et le mouvant, consacrés à définir par quelle méthode la philosophie doit s'y prendre pour appréhender le mouvement sans trop le corrompre. Nous ne nous y arrêterons pas car elles correspondent à un usage courant et imagé du langage dont Bergson se sert, ni plus ni moins que chacun d'entre nous. 
traverse sans jamais pouvoir être possédé ni même vraiment saisi. Pour sortir de ce type d'aporie, il nous faut comprendre la nature et l'orientation de l'indéterminé que sous-tend cette ombre du regard. En effet, chez Bergson, il ne fait aucun doute que cet indéterminé est l'expression privilégiée d'une irréductible liberté; une liberté dont il nous faut comprendre toute la signification ontologique et qui ne saurait, par exemple, se réduire à la figure d'une liberté exprimée dans le jugement telle que la définit Alain.

Nous nous trouvons ainsi confrontés à un paradoxe, puisque c'est à une incapacité, à savoir notre incapacité à traduire pleinement le réel en concepts, que nous devons la part la plus authentique de notre liberté. Ce paradoxe, qui est décisif dans l'œuvre de Bergson, nécessite encore quelques explications.

En premier lieu, et c'est là l'essentiel, pour comprendre l'œuvre de Bergson il faut toujours conserver en mémoire ce qu'il écrit dans l'avant-propos de l'Essai sur les données immédiates de la conscience. Cette page, partagée en deux paragraphes très courts, introduit toute son œuvre dans la mesure où il y pose de façon explicite l'intentionnalité de sa recherche. Le premier paragraphe, de facture nominaliste, pose d'emblée la question des limites du langage qui déplace les problèmes inhérents à la vie plutôt qu'il ne parvient à les résoudre. Le second paragraphe quant à lui explique quelle est la question posée par l'ouvrage. Cette question, présente dans chacun de ses essais, et ceci jusqu'au controversé Deux sources de la morale et de la religion, porte sur les conditions d'expression de la liberté. Il y écrit ainsi : «Nous avons choisi, parmi les problèmes, celui qui est commun à la métaphysique et à la psychologie, le problème de la liberté ${ }^{31}$.»

On pourrait ainsi dire que si la durée et l'ontologie du mouvement constituent les résultats de la philosophie bergsonienne, si l'intuition en est l'esprit plutôt que la méthode, elle est portée par une intentionnalité forte qui fait que, la vie humaine, de l'évolution biologique qui l'a forgée jusqu'à l'inscription sociale des comportements, n'a de sens que dans l'expression d'une indétermination qui est le propre de la liberté. La conclusion de ce même Essai est, à ce titre, particulièrement significative et nous donne à voir un autre plan d'interprétation pour l'ombre, cette fois-ci ni propre ni figurée, mais pleine de cette négativité dont jusqu'ici nous n'avons cessé de chercher à montrer qu'elle n'avait pas sa place dans la philosophie de Bergson.

31. Henri Bergson, Essai sur les données immédiates de la conscience, p. 3. 
Ainsi, avant de s'en prendre, une fois encore, à ce qu'il nomme «l'erreur de Kant», Bergson introduit, sans la nommer explicitement, la notion d'«attention à la vie» dont il sera question dans Matière et mémoire et par laquelle passe une réelle appréhension de la durée.

Mais ces moments où nous nous ressaisissons ainsi nous-mêmes sont rares, et c'est pourquoi nous sommes rarement libres. La plupart du temps, nous vivons extérieurement à nous-mêmes, nous n'apercevons de notre moi que son fantôme décoloré, ombre que la pure durée projette dans l'espace homogène ${ }^{32}$.

Nous sommes effectivement ici confrontés à une pure négativité. La différence est que, contrairement au négatif posé par la dialectique, elle ne tient pas à la vie, mais au risque que chacun de nous court de ne pas mener sa vie. Cette négativité n'est donc en aucune façon un destin et ne relève pas de la métaphysique. Elle n’a pas de fonction historique, ne renvoie en rien sur la figure classique de la nécessité, mais relève pleinement d'un domaine qui est celui de l'aliénation. Elle est une pure négativité pour la simple raison qu'elle ne produit rien de positif, pas même un début d'expérience, dans la mesure où toute expérience induit, pour advenir, fût-ce de façon hypothétique, ce minimum d'attention réflexive dont précisément l'aliénation nous interdit l'accès.

Ce n'est ni le propre ni le figuré de l'ombre - autrement dit ni l'ombre de notre corps ni l'ombre de la représentation - qui portent en elles-mêmes le germe de cette aliénation. Cette dernière est engendrée par tout ce qui, dans la vie matérielle, sociale, affective (la liste ne saurait être exhaustive), contribue à nous placer dans la position d'un automate. La «vie mécanique », c'est-à-dire la vie menée comme un simple mécanisme d'autoreproduction, voici la vraie négativité. Et c'est effectivement une négativité qui ne retranche pas mais, copie après copie, surajoute du même là où ne devrait apparaître que du mouvement et de l'inédit ${ }^{33}$. Comme Bergson l'exprime dans L'énergie spirituelle, après avoir émis l'hypothèse qu'il puisse être lui-même un automate dont la nature échapperait à son auditoire ${ }^{34}$, c'est un risque permanent consistant à laisser la matière ${ }^{35}$ s'enrouler autour de la conscience, la plier à son propre

32. Henri Bergson, Essai sur les données immédiates de la conscience, p. 151.

33. Nietzsche ne fait pas partie des références de Bergson, et pourtant on ne peut s'empêcher de trouver ici, entre eux, une singulière communauté d'esprit.

34. Henri Bergson, L'énergie spirituelle, p. 819.

35. Au sens d'une matière dont il dit dans «Le possible et le réel » qu'elle est une constante répétition. (Henri Bergson, «Le possible et le réel» [1930], La pensée et le mouvant, p. 1332) 
automatisme, l'endormir enfin de sa propre inconscience ${ }^{36}$. C'est un «piège », nous dit-il, ce qui revient à affirmer que sa pleine connaissance doit nous permettre d'y échapper et que nul déterminisme ne saurait justifier que nous nous y laissions prendre. Voilà même ce qui doit constituer pour la philosophie son projet permanent: à savoir nous armer contre ce piège d'une vie mécanique! Au demeurant, suggère Bergson, qu'est-ce que l'esprit, sinon une conscience qui a échappé au piège de l'automatisme, ou, plus loin encore si l'on remonte l'histoire de l'évolution, une «matière qui s'est transformée pour acquérir de la liberté37. »

En second lieu, et c'est ici que réside l'essence de ce paradoxe, c'est en raison même de l'ombre qui réside dans notre regard que nous pouvons échapper au piège de l'aliénation mécanique. Nos concepts doivent leur qualité d'ombre au fait qu'ils sont toujours en décalage par rapport au réel : dans l'excès si l'on considère la sensation dont ils cherchent à faire un état stable; par défaut vis-à-vis de la perception dont ils croient rendre compte en se limitant à en recevoir quelques bribes. C'est néanmoins à cette série de décalages que nos concepts doivent d'être les agents de cette liberté, inouïe, que nous nous accordons dans l'acte de représentation. Leur imperfection constitue assurément leur plus belle qualité. Encore nous faut-il éviter de tomber dans le piège du dogmatisme, qui n'est jamais qu'un automatisme singulier par lequel les concepts s'appliquent à eux-mêmes cette interruption du mouvement pour laquelle ils sont nés et dont se sert, en chaque instant, la représentation.

C'est de cette liberté dont parle Bergson, par exemple, lors des travaux de la société française de philosophie ${ }^{38}$, où il fut amené à répondre à André Lalande $^{39}$, dont le projet était précisément de conférer aux principaux concepts employés par la philosophie une acception définitive. La réponse de Bergson

36. Henri Bergson, L'énergie spirituelle, p. 829.

37. Henri Bergson, L'énergie spirituelle, p. 830-831.

38. Lors de la séance du 23 mai 1901. (Henri Bergson, «Discussion à la société française de philosophie», dans Mélanges, Paris, Presses universitaires de France, 1972 [1885-1941], p. 502-505)

39. Philosophe particulièrement représentatif de la troisième république, de sa morale jusqu'à son rationalisme empreint d'un quiétisme universaliste, André Lalande a mené pendant plus d'un quart de siècle ce travail de lexicographe qui se concrétisa par la publication du Vocabulaire technique et critique de la philosophie, Paris, Éditions Felix Alcan, 2 volumes, 1926. 
est sur ce point particulièrement significative de cette liberté acquise et préservée dans l'indéterminé des concepts.

Est-il utile d'arrêter définitivement la signification des termes là où les idées sont encore flottantes? Peut-on fixer le sens du mot quand on discute encore sur la nature de la chose? Doit-on poser des définitions de mots qui seront nécessairement des solutions anticipées, et l'indécision du mot n'a-t-elle pas quelquefois pour avantage de rappeler que la question reste ouverte ${ }^{40}$ ?

Le ton, comme toujours chez Bergson, est mesuré. Il n'en demeure pas moins que la condamnation de l'entreprise est totale. Les mots de la métaphysique, ajoute-t-il plus loin, doivent rester des «énoncés de problèmes ${ }^{41}$ ». Ils ne doivent pas se travestir en un réel dont l'illusion serait d'autant plus funeste qu'elle parviendrait à faire oublier les mécanismes dont elle est issue. À l'image des marionnettes de Christian Boltanski dont il a été question plus haut, les ombres portées par les concepts peuvent couvrir toute la surface du mur et, ce faisant, produire en nous un sentiment d'étonnement, de crainte ou d'admiration, l'essentiel est que rien du dispositif ne soit caché; autrement dit que l'artifice demeure présent à la conscience de chacun comme artifice, représentation éphémère de l'ombre, efficiente, celle-ci, qui anime notre regard.

En dernier lieu, c'est sous la protection de ce regard, porteur d'une ombre qui réduit à néant l'opposition stérile entre objectivité et subjectivité, que la liberté parvient parfois à se frayer un chemin vers ce partage équivoque du sensible qu'est l'œuvre d'art. Sur un plan littéral déjà, dans la mesure où, tel que Bergson le présente dans Le rire, l'élan esthétique se définit comme ce qui rompt, non pas avec le réel, mais avec toute reproduction de ce réel qui soit portée par un sentiment d'utilitét2. Un renoncement à l'utilité dont il nous dit,

40. Henri Bergson, «Discussion à la société française de philosophie», p. 503.

41. Henri Bergson, «Discussion à la société française de philosophie», p. 503. Il ajoute certes à ceci, un «pour le moment» qui tend à laisser penser que son opposition n'est pas une opposition de principe mais repose sur des considérations pragmatiques. Mais lorsque Lalande lui répond en affirmant que les termes de ce problème doivent bien, eux, être dotés d'une définition précise, Bergson réfute point par point l'exemple donné par son interlocuteur, montrant par là que même la multiplicité des points de vue ne saurait donner le moindre aperçu de ces sens intermédiaires dont il dira dans La pensée et le mouvant que c'est en eux que se trouve l'expression du mouvement.

42. Henri Bergson, Le rire. Essai sur la signification du comique [1900], dans (Euvres, p. 396. 
dans L'énergie spirituelle, qu'il est porté par un affect, en l'occurrence la joie, plutôt que par cet état de réalisation qu'est le plaisir ${ }^{43}$ et dont nous voyons bien, aujourd'hui, à quel point il peut donner lieu à l'inscription d'un quotient économique. De ce choix de l'affect contre l'état, il ressort principalement que le seul absolu dont peut se revendiquer l'œuvre d'art est son inachèvement, c'està-dire la permanence du mouvement auquel elle est soumise et qui nous permet d'approcher au plus près la vie. Le paradoxe trouve même ici une nouvelle formulation puisque, pour y parvenir, il faut disposer d'un «sens ou d'une conscience moins adhérents à la vie», c'est-à-dire qui soient détachés d'une perception dont la tâche est d'effectuer un tri préalable parmi les ombres que le regard lui impose. "C'est parce que l'artiste songe moins à utiliser sa perception qu'il perçoit un plus grand nombre de choses ${ }^{44}$.

L'ombre dans le regard tend dès lors à se traduire par un regard de l'ombre. Regard propre à s'arrêter sur les ombres, à les façonner, à les investir comme autant de places fortes de ce monde où il est si tentant de n'être qu'un automate. Regard de l'ombre à propos duquel la philosophie, qui fait se mouvoir les concepts sur le mur de nos questions sans réponses, doit se réjouir d'être toujours mise en échec. Si donc, lorsqu'il fait l'éloge de Ravaisson ${ }^{45}$, Bergson défend la thèse d'une complémentarité de l'art et de la métaphysique, cela tient effectivement à un renversement total de cette fonction du regard qui, dans les deux cas, introduit une distance - ou plutôt une tension - entre la perception et la représentation. Ainsi, si «l'art est une métaphysique figurée » et "la métaphysique une réflexion sur l'art», cela tient autant à leur écart quant à la relation que l'un et l'autre entretiennent avec le logos, qu'à la disposition non utilitaire du regard qui les supporte tous deux et les investit d'une liberté en nulle autre instance égalée.

Une maxime chinoise dit qu'au centre du monde, il y a un arbre auprès duquel rien ne donne écho, auprès duquel rien de ce qui est parfaitement droit ne donne d'ombre ${ }^{46}$. La philosophie de Bergson, dans son refus de la dialecti-

43. Henri Bergson, L'énergie spirituelle, p. 832.

44. Henri Bergson, «La perception du mouvement » [1911], La pensée et le mouvant, p. 1373 .

45. Henri Bergson, «La vie et l'œuvre de Ravaisson» [1904], La pensée et le mouvant, p. $1450-1481$.

46. Maxime relevée par Marcel Granet, La pensée chinoise, citée par Simone Weil, "Cahiers XII », dans Euvres complètes, tome VI, vol. 3, Paris, Éditions Gallimard, 1991 [1942], p. 530. 
que, tend ainsi à nous rappeler que la seule rectitude possible est celle des automates et que l'ombre, comme l'écho, participent d'un flux constant de la vie où la négativité n'est jamais qu'une représentation parmi d'autres, une façon comme une autre de figer le présent et, ce faisant, de le laisser échapper. 